\title{
ÍNDICE DE AUTORES
}

Volume 5 - 2012

A

ALMEIDA, E. R. de

ALMEIDA, M. V. de

AMARAL, F. M.

ANDALÉCIO, A. M. L.

ANDRUKIU, A. M. G.

B

BOMFIM, N. R.

BRONZE JÚNIOR, J. C. F.

C

CARVALHO, $\mathrm{P}$.

CORRÊA, C. H. W.

COSTA, E. R. C.

F

FAGUNDES, A. F. A.

FARIAS, M. F. de

FLECHA, A. C.

FRAIZ BREA, J. A.

FRAIZ GARCÍA, A.

G

GÂNDARA, J. M. G.

GÂNDARA, J. M. G.

GOMES, B. M. A.

GOMES, D. S.
O

527 OGANAUSKAS, D. S. N. 563

340 OLIVEIRA, M. L. S. 164

104

$527 \quad \mathbf{P}$

563 PERINOTTO, A. R. C. 82

PÍCCOLO, D. R. $\quad 466$

PINHEIRO, R. C. dos S. 275

252 PIROLO, M. C. F. 507

489

$\mathbf{R}$

REIS, A. M. da S. 252

545 RIBAS, L. C. C. 391

19 ROCHA, F. G 124

310 ROQUE, A. 545

$\mathbf{S}$

489 SAMPAIO, D. O. 489

104 SANTOS, C. A. de J. 275

443 SILVA, S. N. da 209

423 SOUSA, C. V.e 489

423 SOUZA, M. de 231

$\mathbf{T}$

183 TEIXEIRA, A. R. 231

466 TELLES, D. H. Q. 183

563 TORRES, R. G. 507

82 
I

INÁCIO, R. de O.

K

KORSTANJE, M. E.

KUNZ, J. G.

L

LARA, J. E.

LOPES, E. R.

M

MARACAJÁ, K. F. B.

MATIAS, $\mathrm{M}$.

MELO, R. de S.

MOTA, K. M.

MUNDET, L.
V

443 VILA, N. A.

VIANA, M. F.

$368 \quad$ W

144 WITTMANN, M. L.

$\mathbf{X}$

489 XAVIER, T. R.

443

295

Z

ZUCULOTO, J. R. M.

391
104

58

209

82

584 